\title{
Evaluation of Ursolic Acid as the Main Component Isolated from Catharanthus Roseus against Hyperglycemia
}

\author{
Nguyen Thanh Tam ${ }^{1}$, Dao Duc Thien ${ }^{1}$, Tran Van Sung ${ }^{1}$, \\ Nguyen Thi Hoang Anh ${ }^{1}$, Trinh Thi Thuy ${ }^{1}$, Khuat Huu Trung ${ }^{2}$, \\ Tran Dang Xuan ${ }^{3}$, Tran Dang Khanh ${ }^{2 *}$ \\ ${ }^{1}$ Institute of Chemistry, Vietnam Academy of Science and Technology, Hanoi, Vietnam; \\ ${ }^{2}$ Department of Genetic Engineering, Agricultural Genetics Institute, Tuliem, Hanoi, Vietnam; \\ ${ }^{3}$ Department of Development Technology, Graduate School for International Development and \\ Cooperation (IDEC), Hiroshima University, Japan
}

E-mail address: tdkhanh@vaas.vn

Keywords: Catharanthus roseus, anti-glucosidase, diabetic, anti-hyperglycemia

\begin{abstract}
Ursolic acid with large amount (0.67\% of dried plant weight) along with 7 compounds, namely as spatozoate (1), kaurenoic acid (2), ursonic acid (3), 3-hydroxy-11-ursen28,13-olide (4), ursolic acid (5), vindoline (6) and mixture of $\beta$-sitosterol and stigmasterol were isolated from dichloromethane and ethyl acetate extracts which have shown anti-glucosidase activity of the whole plant of C.roseus. Some isolated compounds and their derivatives were also tested for anti-glucosidase and cytotoxicity.Ursolic acid was examined for hypoglycemic activity in alloxan-induced diabetic mice with dose of 200 and $300 \mathrm{mg} / \mathrm{kg} /$ day, respectively. The results have shown that the blood glucose level were reduced by $45.75 \%$ and $51.31 \%$ to compare with the control group. This study has confirmed that the main component of Vietnamese C. roseus has had significant anti-hyperglycemia activity.
\end{abstract}

\section{INTRODUCTION}

Catharanthus roseus (L.) is an important prerennial tropical plant belonging the Apocynaceae family, comsists of eight species, seven endemic to Madagasca (C.coriaceus, C. lanceus, C.longifolius, C.ovalis, C.roseus, C. scitulus, C.trichophyllus) and one, C.pusillus from India. Among them, $C$. roseus is widely used to treat with the numerous fatal human diseases because of its producing a wide spectrum of secondary metabolites, include monoterpenoids, glucosides, steroids, phenolics, flavanoids, anthocyanins and more than 130 different terpenoid indole alkaloids, which showed strong and important pharmacological activities [3, 22].

Much worldwide research attention has been paid in C.roseus as a model for medicinal plant improvement. Traditionally, $C$. roseus is being used for relieving muscle pain, depression of the central nerous system and wasps stings. It is also used to treat many cases of nose bleed, bleeding gums, mouth ulcer, sore throats, internal loss of memory, hypertention, cystitis, gastritis, enteritis, diarrhoea and the rised blood sugar levels. In Vietnam, C. roseus is used in the folk medicine for the treatment of fever, dialysis, skin diseases, and diabetes (Ho 2000). Hypoglycemia is one of the most noticeable activities of $C$. roseus. It has been also used for people with diabetes in South Africa, Australia, Philippine, South Vietnam and the UK. There have been some reports on the hypoglycemic activity of different parts of this plant $[4,12,14]$.

Rapidly economic growth in the developed and developing countries has caused some severe human diseases, especially in diabetes. Currently, there are over 150 million diabetic patients worldwide and expected to increase to 300 million or more by the year 2015 [12]. In the big cities of Vietnam, approximately about $4 \%$ of the population have suffered from diabetes which have been equivelent with 3.3 million cases of diabetes in Vietnam in 2014 [5]. Its complications can cause of many diseases as cardiovascular disease, stroke, chronic kidney failure, foot ulcers, and damage to the eyes. Some chemical constituents of this species have been shown high 
hypoglycemic activity such as vindoline, tetrahydroalstonine, catharanthine, lochericine, leurosine, vindolinine, vindogentianine, ursolic acid [9,21].

In the present study, we have reported that some constituents were isolated from the whole $C$. roseus which have exhibited anti-glucosidase activity, cytotoxicity. This study has also affirmed that ursolic acid is the main hypoglycemic component of $C$. roseus growing in Vietnam.

\section{MATERIALS AND METHODS}

\section{Plant material}

The whole plant of C.roseus was collected in Hoai Nhon, Binh Dinh Province, in December 2012, and was identified by Mr. Nguyen The Anh, Institute of Chemistry, Vietnam Academy of Science and Technology. A voucher specimen No.005 has been kept at $6^{\circ} \mathrm{C}$ in freezer in laboratory of Department of Organic Synthesis, Institute of Chemistry, Vietnam Academy of Science and Technology (VAST).

\section{METHODS}

\section{General}

ESI-MS: UPLC/MS/MS Xevo-TQMS. NMR: Bruker Avance $500 \mathrm{MHz}$. Column chromatography (CC): silica gel $(70-230$ and $230-400$ mesh, Merck). Thin layer chromatography (TLC): DC-Alufolien $60 \mathrm{~F}_{254}$ (Merck). Yeast $\alpha$ glucosidase, p-nitrophenyl- $\alpha$-Dglucopyranoside (pNPG) and alloxan monohydrate were purchased from Sigma.

\section{Extraction and isolation}

The dried and powdered C. roseus of whole plant $(2.2 \mathrm{~kg})$ were extracted with solvents of increasing polarity: $n$-hexane, dichloromethane, ethyl acetate and methanol, successively. The solvent was concentrated under vacuum to give $35.7 \mathrm{~g} n$-hexane-, $23.5 \mathrm{~g}$ dichloromethane, $10.8 \mathrm{~g}$ ethyl acetate and $72.2 \mathrm{~g}$ methanol extracts. The dichloromethane extract was chromatographed on silica gel column, eluting with increasing amount of $\mathrm{MeOH}(0-20 \%)$ in $\mathrm{CH}_{2} \mathrm{Cl}_{2}$ to yield 10 fractions, which were combined according to TLC monitoring. The fraction 2 was rechromatographed over flash silica gel column eluting with $n$-hexane: EtOAc (9.5: 0.5) afforded 8mg spatozoate (1). $10 \mathrm{mg}$ kaurenoic acid (2) was obtained by re-purification of the fraction 3 $(82 \mathrm{mg})$ on silica gel column ( $n$-hexane: EtOAc $=9: 1)$. The fraction $5(315 \mathrm{mg})$ was further purified on the silica gel column eluted with $n$-hexane: EtOAc (9:1) to give $12 \mathrm{mg}$ ursonic acid (3) and a mixture of $\beta$-sitosterol and stigmasterol (ratio of 2:1). The NMR spectral data of $\beta$-sitosterol and stigmasterol are in good agreement with those in reported literature (Suparb and Amorn 1991), and the ratio was determined based on signal integration of $\mathrm{H}-6, \mathrm{H}-22$ and $\mathrm{H}-23$ on their ${ }^{1} \mathrm{H}$ NMR spectra. The NMR spectral data are in good agreement with those in the previous report (Suparb and Amorn 1991). The fraction 6-9 were rechromatographed over flash silica gel column eluting with $\mathrm{CH}_{2} \mathrm{Cl}_{2}: \mathrm{MeOH}$ (93:7) to give $15 \mathrm{mg}$ 3-hydroxy-11-ursen-28,13-olide (4) and 6.8g ursolic acid (5). $10.8 \mathrm{~g}$ ethyl acetate extract was purified on silica gel column eluted with $\mathrm{CH}_{2} \mathrm{Cl}_{2}: \mathrm{MeOH}$ (93:7) to provide $102 \mathrm{mg}$ vindoline $(6)$ and $8 \mathrm{~g}$ ursolic acid.

\section{Assay of cytotoxic activities using the cell lines: HepG2, KB, LU-1, MCF7, SK-Mel2, HL60, SW626, LNCaP and HT29 \\ Cell culture}

The monolayer cancer cell lines (HepG2, KB, LU-1, MCF7, SK-Mel2, HL-60, SW626, LNCaP and HT29) were grown in Dulbeco's modified Eagle medium (DMEM) with 2 mM Lglutamine, $10 \mathrm{mM}$ HEPES, $1.0 \mathrm{mM}$ sodium pyruvate, and 10\% fetal bovine serum - FBS (GIBCO). The cells were subcultured every 3-5 days with the ratio of $(1: 3)$ and incubated at $37^{\circ} \mathrm{C}$ under humidified atmosphere of $5 \% \mathrm{CO}_{2}$. 


\section{Cytotoxic assay}

The above mentioned human cancer cell lines are supplied by Prof. Dr. J. M. Pezzuto, Hawaii University, US and Prof. Jeanette Maier, Milan University, Italy in 2013. The five isolates were evaluated for their cytotoxic activities against seven (except HL60) human cancer cell lines according to the method described by Monk et al [10] and against HL60 cell line according to the method MTT [8]. The cytotoxic potential was assessed by determining the amount of sulforhodamine B (SRB) bound to proteins and was performed in a microtiter plate. The test samples were examined over a concentration range of $0.8-100 \mu \mathrm{g} / \mathrm{mL}$. DMSO $(10 \%)$ was used as the negative control. Ellipticine (Sigma) served as the positive control and examined at the concentration of 10, 2, 0.4 and $0.08 \mu \mathrm{g} / \mathrm{mL}$, respectively. Experimental cultures were plated in microtiter plates (Costar, USA) containing $20 \mu \mathrm{L}$ of each test sample and $180 \mu \mathrm{L}$ of growth medium $(10 \%$ FBS $)$ per well at density of 6000 cells/well. The duration assay was adopted as 3 days. Test plates were incubated in a humidified atmosphere of $5 \% \mathrm{CO}_{2}, 37{ }^{0} \mathrm{C}$ for $72 \mathrm{~h}$, while the 0 -day control was incubated for $1 \mathrm{~h}$. After incubation, cells were fixed for $30 \mathrm{~min}$. to the plastic substratum by the addition of $100 \mu \mathrm{L}$ of cold $20 \%$ aqueous trichloroacetic acid (TCA) for at least $1 \mathrm{~h}$ at $4^{0} \mathrm{C}$. Fixed cells were then stained with $0.4 \% \mathrm{SRB}(\mathrm{w} / \mathrm{v})$ dissolved in $1 \%$ acetic acid and washed four times with $1 \%$ acetic acid. The bound dye was then solubilized by the addition of $10 \mathrm{mmol}$ unbuffered Tris base (Sigma), absorption was measured at $515 \mathrm{~nm}$ with a microplate reader (BioRad). All the experiments were performed three times with the mean absorbance values calculated.

Growth, expressed as a percentage of the negative control, was calculated with the equation (OD: Optical Density):

$$
\begin{aligned}
& \% \text { growth }=\frac{O D(\text { test substance })-\text { OD }(\text { day } 0) \times 100}{\text { OD }(\text { negative control })-\text { OD }(\text { day } 0)} \\
& \% \text { inhibition }=100 \%-\% \text { growth }
\end{aligned}
$$

\section{MTT method}

The HL60 cell growth inhibition was determined by MTT assay. After incubation for $72 \mathrm{~h}$, the media was removed and the cells were incubated with $20 \mu \mathrm{L}$ MTT $(5 \mathrm{mg} / \mathrm{mL})$. After incubation for $4 \mathrm{~h}$ at $37^{\circ} \mathrm{C}$ in an atmosphere of $5 \% \mathrm{CO}_{2}$ incubator, the formazan crystal formed were dissolved by adding $100 \mu \mathrm{L} /$ well of DMSO. The optical density was measured at $515 \mathrm{~nm}$ wave length with ELISA Plate Reader (Bio-Rad) equipment. The number of viable cells was proportional to the extent of formazan production. Growth, expressed as a percentage of the negative control, was calculated with the equation:

$$
\begin{aligned}
& \% \text { growth }=\frac{\text { OD (test sample }) \times 100}{\text { OD (negative control) }} \\
& \% \text { inhibition }=100 \%-\% \text { growth }
\end{aligned}
$$

\section{$\alpha-G l u c o s i d a s e$ inhibitory activity in vitro}

$\alpha$-Glucosidase inhibitory activity was performed according to the method of Afrapoli-Moradi et al (2012). $\alpha$-Glucosidase inhibitory activity was determined spectrophotometrically in a 96-well plate based on p-nitrophenyl- $\alpha$-D-glucopyranoside (pNPG) as the substrate. The tested samples were dissolved in DMSO and added phosphate buffer $10 \mathrm{mM}(\mathrm{pH} \mathrm{6.8)}$. The mixture was putted in a 96-well plate at various concentrations as $1000 \mu \mathrm{g} / \mathrm{ml}, 500 \mu \mathrm{g} / \mathrm{ml} ; 100 \mu \mathrm{g} / \mathrm{ml} ; 20 \mu \mathrm{g} / \mathrm{ml} ; 4 \mu \mathrm{g} / \mathrm{ml}$. $20 \mu 1$ $\alpha$-glucosidase $(0,5 \mathrm{U} / \mathrm{ml})$ and $120 \mu 1$ phosphate buffer $100 \mathrm{mM}(\mathrm{pH} 6.8)$ were added each well, mixed and incubated at $37^{\circ} \mathrm{C}$ for $15 \mathrm{~min}$. The reaction was processed at $37^{\circ} \mathrm{C}$ for $60 \mathrm{~min}$ and stopped by adding $80 \mu \mathrm{L}$ of $0.2 \mathrm{M}$ sodium carbonate solution. Absorbance of the wells was measured with a microplate reader at $405 \mathrm{~nm}$. The reaction system without plant extracts was used as control (mixture of DMSO 10\%, phosphate buffer, enzyme and pNPG were used as the control). The 
system without $\alpha$-glucosidase was used as blank (mixture of tested sample, phosphate buffer and pNPG were used as blank). Acarbose was used as positive control. Each experiment was conducted in triplicate. The enzyme inhibitory rates of samples were calculated as follows:

$$
\begin{gathered}
\text { Inhibition }(\%)=\left(1-\mathrm{A}_{\text {Test sample }} / \mathrm{A}_{\mathrm{Control}}\right) * 100 \\
\text { In which: } \mathrm{A}_{\text {Control }}=\mathrm{OD} \text { Control }-\mathrm{OD}_{\text {blank }} \\
\mathrm{A}_{\text {Test sample }}=\mathrm{OD}_{\text {Test sample }}-\mathrm{OD}_{\text {blank mauthu }}
\end{gathered}
$$

$\alpha$-Glucosidase inhibitory activity was recorded spectrophotometrically in a 96-well plate based on pNPG as substrate ( $\mathrm{Li}$ et al. 2011). The assay mixture $(160 \mu \mathrm{L})$ contained $8 \mu \mathrm{l}$ of a sample in DMSO (or DMSO itself as control), $112 \mu$ phosphate buffer (pH 6.8) and $20 \mu \mathrm{L}$ enzyme solution $\left(0.2 \mathrm{U} / \mathrm{ml} \alpha\right.$-glucosidase in phosphate buffer), mixed and incubated at $37^{\circ} \mathrm{C}$ for $15 \mathrm{~min}$, and then, $20 \mu \mathrm{L}$ substrate solution $(2.5 \mathrm{mM}$ pNPG prepared in the same buffer) added. The reaction was processed at $37^{\circ} \mathrm{C}$ for $15 \mathrm{~min}$ and stopped by adding $80 \mu \mathrm{L}$ of $0.2 \mathrm{M} \mathrm{Na} 2 \mathrm{CO} 3$ solution. Amount of the p-nitrophenol released from PNP-glycoside was quantified on a 96 microplate reader at $405 \mathrm{~nm}$. The inhibitory rates (\%) were calculated according to the formula: [1-(sample ODtest- sample ODblank)/ (control ODtest- control ODblank)] $\times 100 \%$. Sample ODtest stand for solution of sample + enzyme + substrate. Sample ODblank stand for solution of sample + buffer. Control ODtest stand for solution of buffer + enzyme + substrate. Control ODblank stand for solution of buffer. All reactions were carried out with three replications. Acarbose was used as positive control.

\section{Hypoglycemic effect assay in vivo}

\section{Experimental animal}

Healthy BALB/c mice were housed in cages under laboratory conditions at standard conditions of temperature, light $\left(12 \mathrm{~h} \mathrm{light/dark}\right.$ cycle, $25^{\circ} \mathrm{C}$ and humidity $\left.45-65 \%\right)$ in Institute of Biotechnology, VAST. The animals were fed with standard rodent diet and water ad libitum.

\section{Experimental design}

Hyperglycemia for 32 mice weighed from 27-32g by injecting alloxan monohydrate according to the method of Yanarday and Colak [24]. The fasting blood glucose was determined after $72 \mathrm{~h}$ injected alloxan monohydrate solution and fasted $12 \mathrm{~h}$ before completion. The blood glucose level greater than or equal to $8 \mathrm{mmol} / \mathrm{L}$ is considered diabetes mellitus [15]. Twenty four out of 32 induced diabetic mice were divided into four experimental groups so that the average blood glucose values in each group is equal. Group 1 diabetic control (DC). Group 2 diabetic rats given acarbose $(50 \mathrm{mg} / \mathrm{kg} /$ day). Group 3 diabetic rats given ursolic acid $(200 \mathrm{mg} / \mathrm{kg} /$ day $)$. Group 4 diabetic rats given ursolic acid (300 mg/kg/day). The animals were treated for 9 days. To analyze the level of postprandial blood glucose, blood samples were collected from the eyes and mice were fasted for 6 $\mathrm{h}$ before the collection of blood samples.

$\%$ blood glucose level increased or decreased compared before experimental.

$\%$ decrease compared to before experimental $=100 \times(\mathrm{G} 1-\mathrm{G} 2) / \mathrm{G} 1$

$\%$ increase compared to after experimental $=100 \times(\mathrm{G} 2-\mathrm{G} 1) / \mathrm{G} 2$

In which: $\quad$ G1: The blood glucose level before experimental

G2: The blood glucose level after experimental

\section{Statistical Analyses}

All the treatments were calculated in a completely randomized design with at least thrice. Data were analyzed using program Microsoft Excel 2013. The $\mathrm{IC}_{50}$ (50\% inhibitory concentration) was determined by plotting concentrations against \% growth using nonlinear regression analysis from Table Curve software 


\section{RESULTS AND DISCUSSION}

\section{Chemical structures of the isolated compounds}

From dichloromethane and ethyl acetate extracts of the whole of $C$. roseus having antiglucosidased, seven compounds: spatozoate (1), kaurenoic acid (2), ursonic acid (3), 3 $\beta$-hydroxy11-ursen-28,13-olide (4), ursolic acid (5), vindoline (6) along with a mixture of $\beta$-sitosterol (7) and stigmasterol (8) with ratio of 2:1 were isolated and determined (Fig 1). This is the first time reported on isolation of compounds $\mathbf{1}, \mathbf{2}, \mathbf{3}$ and $\mathbf{4}$ from C. roseus in Vietnam.

\section{Spatozoate (1)}

ESI-MS m/z: $335.02[\mathrm{M}+\mathrm{Na}]^{+} .{ }^{1} \mathrm{H}-\mathrm{NMR}\left(500 \mathrm{MHz}, \mathrm{CDCl}_{3}\right): \delta 7.75-7.74(1 \mathrm{H}, \mathrm{m}, \mathrm{H}-6), 7.72-$ 7.70 (1H, m, H-3), 7.53-7.51 (2H, m, H-4 \& H-5), 7.42 (2H, br d, $J=7.5$ Hz, H-2'/H-6'), 7.39-7.33 (3H, m, H-3'- H-4'), 5.34 (2H, s, H-8), 4.19 (2H, t, $J=6.5$ Hz, H-2' '), 1.65-1.60 (2H, m, H-3''), 1.41-1.36 (2H, m, H-4'"), 0.93 (3H, t, $\left.J=7.5 \mathrm{~Hz}, \mathrm{H}-5{ }^{\prime \prime}\right) .{ }^{13} \mathrm{C}-\mathrm{NMR}\left(125 \mathrm{MHz}, \mathrm{CDCl}_{3}\right): \delta 167.7(\mathrm{C}-$ 7), 167.4 (C-1"'), 135.6 (C-2), 132.5 (C-1), 131.9 (C-1'), 131.1 (C-5), 130.9 (C-4), 129.0 (C-6), 128.9 (C-3), 128.6 (C-2'/C-6'), 128.4 (C-3'/C-5'), 128.37 (C-4'), 67.5 (C-8), 65.6 (C-2'’), 30.5 (C3''), 19.2 (C-4'’), 13.7 (C-5'').

\section{Kaurenoic acid (2)}

ESI-MS $m / z: 301.17[\mathrm{M}-\mathrm{H}]^{-} .{ }^{1} \mathrm{H}-\mathrm{NMR}\left(500 \mathrm{MHz}, \mathrm{CDCl}_{3}\right): \delta 4.80(1 \mathrm{H}, \mathrm{s}, \mathrm{H}-17 \mathrm{a}), 4.74(1 \mathrm{H}, \mathrm{s}$, $\mathrm{H}-17 \mathrm{~b}), 2.64$ (1H, br s, H-13), 1.24 (3H, s, H-18), 0.95 (3H, s, H-20). ${ }^{13} \mathrm{C}-\mathrm{NMR}\left(125 \mathrm{MHz}, \mathrm{CDCl}_{3}\right)$ :

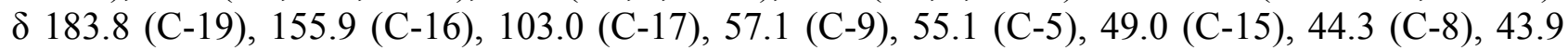
(C-13), 43.7 (C-4), 41.3 (C-1), 40.7 (C-7), 39.7 (C-3), 39.7 (C-10), 37.9 (C-14), 33.1 (C-12), 29.0 (C-18), 21.9 (C-6), 19.1 (C-2), 18.5 (C-11), 15.6 (C-20).

\section{Ursonic acid (3)}

ESI-MS $m / z:: 477.22[\mathrm{M}+\mathrm{Na}]^{+}$(positive ion), $453.26[\mathrm{M}-\mathrm{H}]^{-}$(negative ion). ${ }^{1} \mathrm{H}-\mathrm{NMR}(500$ $\left.\mathrm{MHz}, \mathrm{CDCl}_{3}\right): \delta 5.26(1 \mathrm{H}, t$-like, H-12), 2.58-2.51 (1H, m, H-2a), 2.41- $2.36(1 \mathrm{H}, \mathrm{m}, \mathrm{H}-2 \mathrm{~b}), 2.20$ $(1 \mathrm{H}, \mathrm{d}, J=11.5 \mathrm{~Hz}, \mathrm{H}-18), 1.09\left(3 \mathrm{H}, \mathrm{s}, \mathrm{CH}_{3}\right), 1.08\left(3 \mathrm{H}, \mathrm{s}, \mathrm{CH}_{3}\right), 1.06\left(3 \mathrm{H}, \mathrm{s}, \mathrm{CH}_{3}\right), 1.03(3 \mathrm{H}, \mathrm{s}$, $\left.\mathrm{CH}_{3}\right), 0.96\left(3 \mathrm{H}, \mathrm{d}, J=6.5 \mathrm{~Hz}, \mathrm{CH}_{3}\right), 0.86\left(3 \mathrm{H}, \mathrm{d}, J=6.5 \mathrm{~Hz}, \mathrm{CH}_{3}\right), 0.83\left(3 \mathrm{H}, \mathrm{s}, \mathrm{CH}_{3}\right) .{ }^{13} \mathrm{C}-\mathrm{NMR}$ $\left(125 \mathrm{MHz}, \mathrm{CDCl}_{3}\right): \delta 217.8(\mathrm{C}-3), 183.7$ (C-28), 138.1 (C-13), $125.6(\mathrm{C}-12), 55.3,52.6,48.0,47.4$, $46.8,42.1,39.5,39.3,39.1,38.8,36.7,36.6,34.2,32.5,30.6,28.0,26.6,24.1,23.5,23.4,21.4$, $21.2,19.6,17.0,16.9,15.2$.

\section{$\beta$-sitosterol:stigmasterol (2:1)}

${ }^{1} \mathrm{H}-\mathrm{NMR}\left(500 \mathrm{MHz}, \mathrm{CDCl}_{3}\right): \delta 5.36-5.34(\mathrm{~m}, 1 \mathrm{H}), 5.18-5.13(\mathrm{~m}, 0.36 \mathrm{H}), 5.04-5.02(\mathrm{~m}$, $0.32 \mathrm{H}), 3.56-3.49(\mathrm{~m}, 1 \mathrm{H})$.

$\beta$-sitosterol : ${ }^{13} \mathrm{C}-\mathrm{NMR}\left(125 \mathrm{MHz}, \mathrm{CDCl}_{3}\right): \delta 140.8$ (C-5), 121.7 (C-6), $71.8(\mathrm{C}-3), 56.8,56.1$, 50.2, 45.9, 42.3, 42.3, 39.8, 37.3, 36.5, 36.2, 34.0, 31.9, 31.7, 29.2, 28.3, 26.1, 24.3, 23.1, 21.1 , $19.8,19.4,19.1,18.8,11.8$.

Stigmasterol: ${ }^{13} \mathrm{C}-\mathrm{NMR}\left(125 \mathrm{MHz}, \mathrm{CDCl}_{3}\right): \delta 140.8(\mathrm{C}-5), 138.3(\mathrm{C}-22), 129.3(\mathrm{C}-23), 121.7$ (C-6), 71.8 (C-3), 56.9 (C-14), 56.0 (C-17), 51.2, 50.2, 42.2, 40.5, 39.7, 37.3, 36.2, 35.8, 33.7, 32.4, $31.9,31.5,28.9,25.4,24.4,21.2,21.1,19.4,12.2,12.0$.

\section{3-hydroxy-11-ursen-28,13-olide (4)}

HR-ESI-MS m/z: $477.3339[\mathrm{M}+\mathrm{Na}]^{+} .{ }^{1} \mathrm{H}-\mathrm{NMR}\left(500 \mathrm{MHz}, \mathrm{CDCl}_{3}\right): \delta 5.96(1 \mathrm{H}, \mathrm{dd}, J=10.5$, 10.0, H-11), $5.53(1 \mathrm{H}, \mathrm{dd}, J=10.5,3.5 \mathrm{~Hz}, \mathrm{H}-12), 3.22(1 \mathrm{H}, \mathrm{dd}, J=11.0,4.5 \mathrm{~Hz}, \mathrm{H}-3 \alpha), 2.13(1 \mathrm{H}$, $\mathrm{dt}, J=13.0,6.0 \mathrm{~Hz}, \mathrm{H}-16 \alpha), 1.95(1 \mathrm{H}$, br s, H-9), $1.16(3 \mathrm{H}, \mathrm{s}), 1.05(3 \mathrm{H}, \mathrm{s}), 1.00(3 \mathrm{H}, \mathrm{d}, J=6.5$ $\mathrm{Hz}), 0.99(3 \mathrm{H}, \mathrm{s}), 0.94(3 \mathrm{H}, \mathrm{d}, J=6.5 \mathrm{~Hz}), 0.91(3 \mathrm{H}, \mathrm{s}), 0.79(3 \mathrm{H}, \mathrm{s}) .{ }^{13} \mathrm{C}-\mathrm{NMR}\left(125 \mathrm{MHz}, \mathrm{CDCl}_{3}\right)$ :

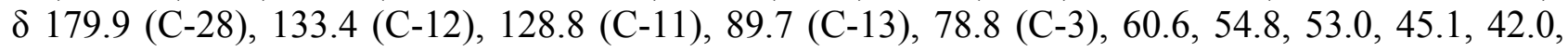
$41.7,40.3,38.9,38.3,38.1,36.4,31.3,31.2,30.8,29.7,27.8,27.0,25.6,22.8,19.2$, 18.9, 17.9, $17.8,17.7,16.1,14.9$. 


\section{Ursolic acid (5)}

ESI-MS $m / z: 479.23[\mathrm{M}+\mathrm{Na}]^{+}$(positive ion), $455.27[\mathrm{M}-\mathrm{H}]^{-}$(negative ion). ${ }^{1} \mathrm{H}-\mathrm{NMR}(500$ MHz, DMSO-d $\left.{ }_{6}\right): \delta 5.12$ (br s, H-12), $4.30(1 \mathrm{H}, \mathrm{d}, J=4.5 \mathrm{~Hz}), 3.01-2.99(1 \mathrm{H}, \mathrm{m}, \mathrm{H}-3), 2.10(1 \mathrm{H}$, d, $J=11.5 \mathrm{~Hz}, \mathrm{H}-18), 1.04$ (3H, s, CH3-27), 0.90 (3H, d, $J=9.5 \mathrm{~Hz}, \mathrm{H}-30), 0.89$ (3H, s, H-23), 0.86 $(3 \mathrm{H}, \mathrm{s}, \mathrm{H}-25), 0.81(3 \mathrm{H}, \mathrm{d}, J=6.5 \mathrm{~Hz}, \mathrm{H}-29), 0.75(3 \mathrm{H}, \mathrm{s}, \mathrm{H}-26), 0.67(3 \mathrm{H}, \mathrm{s}, \mathrm{H}-24) .{ }^{13} \mathrm{C}-\mathrm{NMR}(125$ $\mathrm{MHz}_{\mathrm{CDCl}}$ ): $\delta 178.3(\mathrm{C}-28), 138.2(\mathrm{C}-13), 124.6$ (C-12), 76.9 (C-3), 54.8, 52.4, 47.0, 46.8, 41.6, $39.1,38.5,38.4,38.3,38.2,36.5,36.3,32.7,30.2,28.3,27.5,27.0,23.8,23.3,22.9,21.1,18.0$, $17.0,16.9,16.1,15.2$.

\section{Vindoline (6)}

${ }^{1} \mathrm{H}-\mathrm{NMR}\left(500 \mathrm{MHz}, \mathrm{CDCl}_{3}\right): \delta 6.90(1 \mathrm{H}, \mathrm{d}, J=8.0 \mathrm{~Hz}, \mathrm{H}-14), 6.31(1 \mathrm{H}, \mathrm{dd}, J=8.5,2.5 \mathrm{~Hz}$, $\mathrm{H}-15), 6.09(1 \mathrm{H}, \mathrm{d}, J=2.5 \mathrm{~Hz}, \mathrm{H}-17), 5.86(1 \mathrm{H}, \mathrm{ddd}, J=10.0,4.5,1.5 \mathrm{~Hz}, \mathrm{H}-7), 5.47(1 \mathrm{H}, \mathrm{s}, \mathrm{H}-4)$, $5.24(1 \mathrm{H}, \mathrm{d}, J=10.0, \mathrm{H}-6), 3.80\left(3 \mathrm{H}, \mathrm{s}, \mathrm{COOCH}_{3}\right), 3.79\left(3 \mathrm{H}, \mathrm{s}, \mathrm{OCH}_{3}\right), 3.76(1 \mathrm{H}, \mathrm{s}, \mathrm{H}-2), 3.53-$ $3.49(1 \mathrm{H}, \mathrm{m}), 3.43(1 \mathrm{H}, \mathrm{dt}, J=9.0,4.0 \mathrm{~Hz}), 2.85-2.81(1 \mathrm{H}, \mathrm{m}), 2.69\left(3 \mathrm{H}, \mathrm{s}, \mathrm{NCH}_{3}\right), 2.67(3 \mathrm{H}, \mathrm{s}, \mathrm{H}-$ 19), 2.56-2.51 (1H, m), 2.36-2.31 (2H, m), $2.08\left(3 \mathrm{H}, \mathrm{s}, \mathrm{OCOCH}_{3}\right), 1.68-1.63(1 \mathrm{H}, \mathrm{m}), 1.17-1.13$ $(1 \mathrm{H}, \mathrm{m}), 0.50(3 \mathrm{H}, \mathrm{t}, J=7.5 \mathrm{~Hz}, \mathrm{H}-21) .{ }^{13} \mathrm{C}-\mathrm{NMR}\left(125 \mathrm{MHz}, \mathrm{CDCl}_{3}\right): \delta 171.9\left(\mathrm{OCOCH}_{3}\right), 170.8(-$ $\mathrm{COOCH}_{3}$ ), 161.2 (C-16), 153.7 (C-18), 130.5 (C-6), 125.0 (C-13), 124.1 (C-7), 122.7 (C-14), 104.6 (C-15), 95.8 (C-17), 83.4 (C-2), 79.6 (C-3), 76.4 (C-4), 67.1 (C-19), $55.3\left(\mathrm{Ar}_{-}-\mathrm{OCH}_{3}\right), 52.8(\mathrm{C}-12)$, $52.2\left(\mathrm{COOCH}_{3}\right), 51.9(\mathrm{C}-11), 51.1(\mathrm{C}-8), 44.0(\mathrm{C}-10), 42.9(\mathrm{C}-5), 38.2\left(-\mathrm{NCH}_{3}\right), 30.8(\mathrm{C}-20), 21.0$ $\left(-\mathrm{OCOCH}_{3}\right), 7.6(\mathrm{C}-21)$.
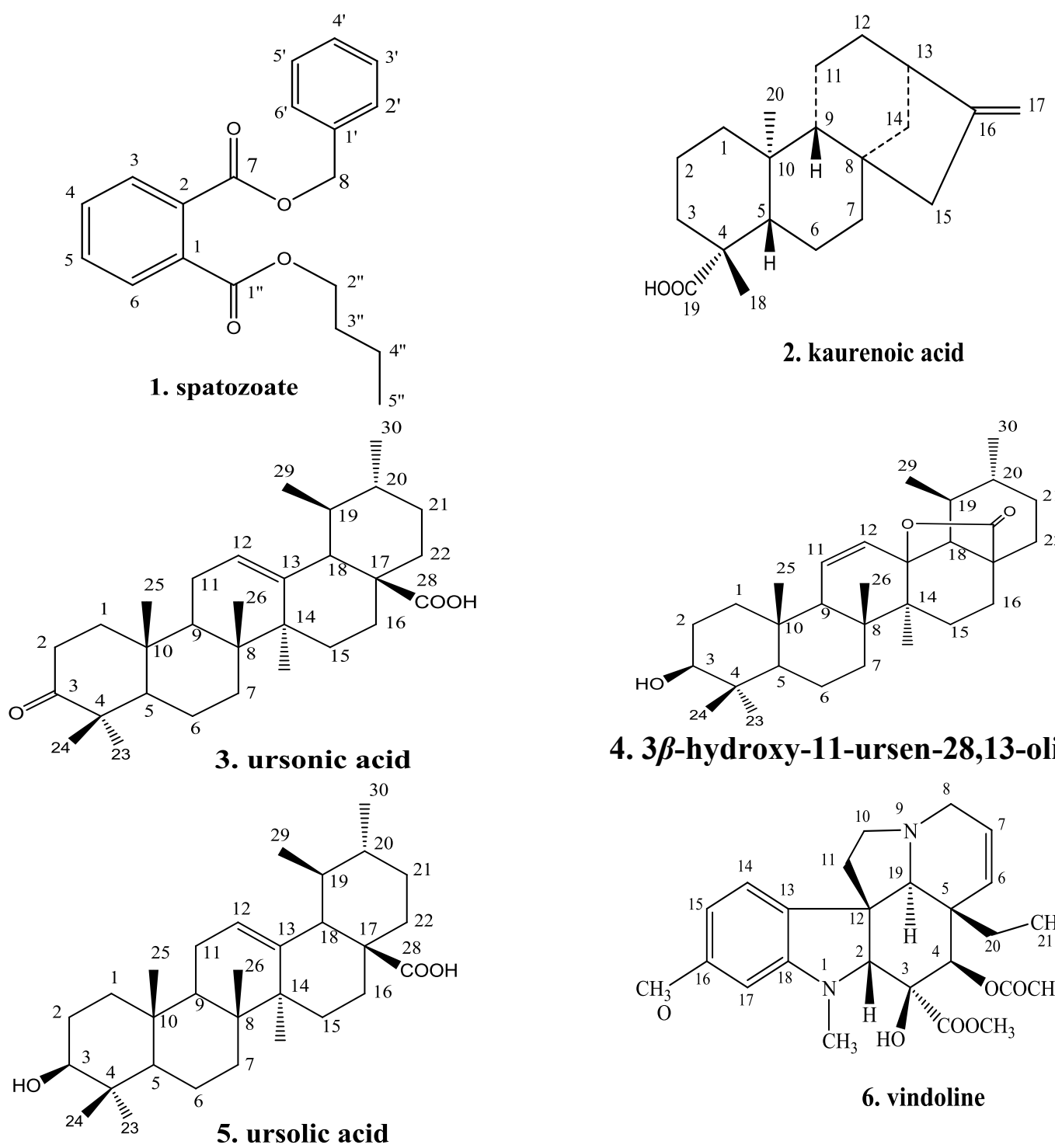

2. kaurenoic acid

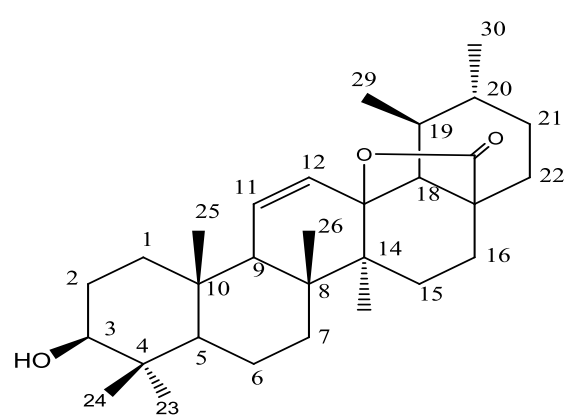

\section{3及-hydroxy-11-ursen-28,13-olide}

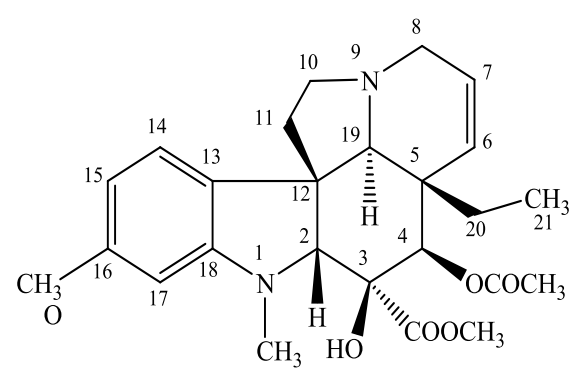

6. vindoline 


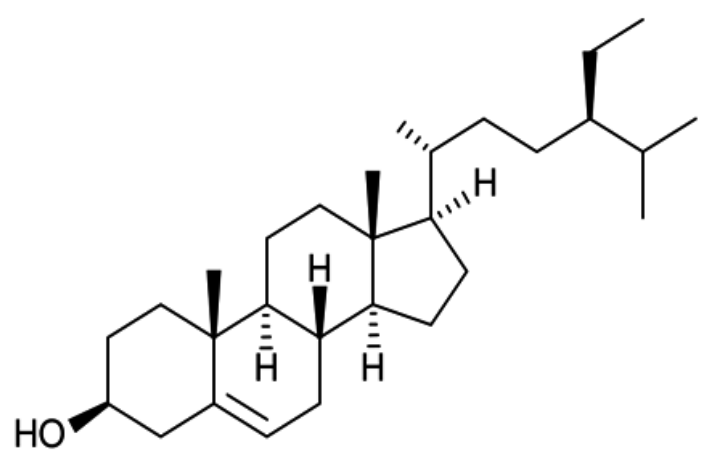

7. $\beta$-sitosterol

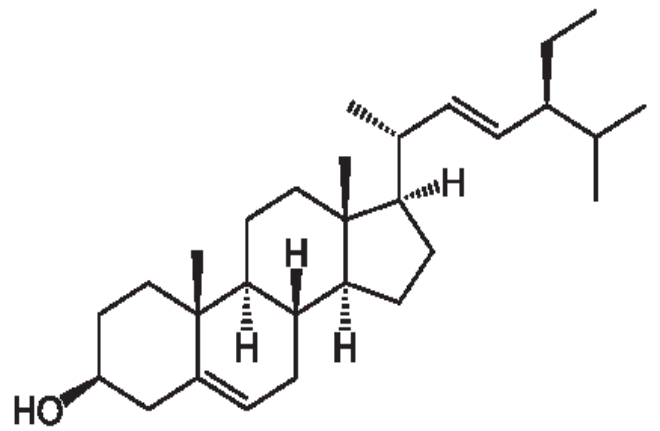

8. stigmasterol

Figure 1. Structures of the compounds isolated from C. roseus

\section{Inhibitory activity against $\alpha$-glucosidase in vitro}

Some isolated compounds have been compounds were tested anti-glucosidase activity. The results have shown that almost the tested compounds have inhibitory activity against $\alpha$-glucosidase, except for compound $\mathbf{2}$. Three compounds 3, 4 and 5 exhibited inhibitory $\alpha$-glucosidase better than acarbose. Especially, ursolic acid (5) is the strongest activity with $\mathrm{IC}_{50}$ value as $3.83 \mu \mathrm{g} / \mathrm{mL}$ (Table 1). This result is in very close agreement with the study of Kang et al [7] whom reported that ursolic acid isolated from EtOAC extract of Osmanthus fragrans disclosed great inhibitory activity of $\alpha$ glucosidase with IC50 $=3.38 \mu \mathrm{g} / \mathrm{mL}$. These results are shown extra evidences to demonstrate for hyperglymecia activity of $C$. roseus.

Table 1. Inhibitory effect of some isolated compounds against $\alpha$-glucosidase.

\begin{tabular}{cc}
\hline Compounds & $\mathbf{I C}_{\mathbf{5 0}}(\boldsymbol{\mu} \mathbf{g} / \mathbf{m L})$ \\
\hline 1 & 282.42 \\
2 & $>500$ \\
3 & 45.92 \\
4 & 92.65 \\
5 & 3.83 \\
Acarbose & 152.56 \\
\hline
\end{tabular}

\section{Cytotoxicity of isolated compounds}

Compounds $1-4$ were tested for cytotoxicity on nine human cancer cell lines: liver cancer (Hep-G2), mouth cancer (KB), lung cancer (LU-1), breast cancer (MCF-7), melanoma cancer (SKMel2), acute leukemia (HL60), ovarian cancer (SW626), androgen-sensitive human prostate adenocarcinoma (LNCaP) and colon adenocarcinoma (HT-29). Their IC50 values are given in the Table 3. Among them, compound 3 was found to be the most active one against all tested cancer cell lines with the $\mathrm{IC}_{50}$ values ranged from 4.36 to $30.15 \mu \mathrm{g} / \mathrm{mL}$. Compounds 2 was found to have moderate cytotoxicity against all nine cell lines with the $\mathrm{IC}_{50}$ in the range of 49.07 to $70.30 \mu \mathrm{g} / \mathrm{mL}$. Compound 4 was inactive on all tested cell lines $\left(\mathrm{IC}_{50}>100 \mu \mathrm{g} / \mathrm{mL}\right)$ (Table 2).

Table 2. Cytotoxic activity of compounds $2-4,7$ and 8

\begin{tabular}{ccccccccccc}
\hline Compounds & & \multicolumn{7}{c}{ In vitro cytotoxicity IC $\mathbf{5 0}(\boldsymbol{\mu g} / \mathbf{m L})$} \\
\hline & Hep G2 & KB & LU-1 & MCF7 & SK- & HL60 & SW626 & LNCaP & HT- \\
1 & & & & & Mel2 & & & & 29 \\
2 & 58.37 & 70.30 & 61.77 & 52.07 & 49.07 & 52.06 & 50.78 & 39.55 & 56.27 \\
3 & $4.36^{*}$ & $5.31^{*}$ & $19.79^{*}$ & $25.36^{*}$ & 24.23 & 14.80 & 33.78 & 12.19 & 30.15 \\
4 & $>100$ & $>100$ & $>100$ & $>100$ & $>100$ & $>100$ & $>100$ & $>100$ & $>100$ \\
Ellipticine & 0.42 & 0.49 & 0.44 & 0.42 & 0.42 & 0.43 & 0.37 & 0.44 & 0.34 \\
\hline
\end{tabular}

*. $\mathrm{IC}_{50}$ of compound 3 on four cell lines Hep G2, KB, LU-1 and MCF7 were repored on by Thien et al [20]. 
Some previous works on evaluating of cytotoxic of $C$. roseus have been conducted. Admad et al [1] reported that crude aqueous extract of C.roseus exhibited differential effects of inhibiting the proliferation of the Jurkat cell line in time-and dose-dependent manner. It is possible that the chemical pathways between the major active compounds may act in concert to suppress Jurkat cells proliferation. The previous report of Siddiqui et al [17] revealed that chroroform fraction extract of C. roseus showed the highest cytotoxic activity against HCT-16 colorectal carcinoma cell line. Some compounds isolated from $C$. roseus such as catharanthine, vindoline showed high cytotoxic activity.

\section{Inhibitory activity against $\alpha$-glucosidase in vitro of the derivatives of ursolic acid}

Ursolic acid (UA) is a pentacyclic triterpenoid which are available in many herbs and fruits. In this study, ursolic acid was isolated from the whole of $C$. roseus with large amount $(0.67 \%$ of dried plant weight). In previous research, we have successfully synthesized a series of ursolic acids which was listed in the Table 3 (Thien et al. 2013). In order to find out their derivatives as hypoglycemic agents, we have further tested anti-glucosidase activity of ursolic acid's derivatives

Table 3. Inhibitory effect of derivatives of ursolic acid against $\alpha$-glucosidase

\begin{tabular}{|c|c|}
\hline Compounds & $\mathrm{IC}_{50}(\mu \mathrm{g} / \mathrm{mL})$ \\
\hline $3 \beta$-Acetoxy-urs-12-ene-28-oic acid & 9.08 \\
\hline $3 \beta$-Succinoyl-urs-12-en-28-oic acid & 125.07 \\
\hline $3 \beta$-phthaloyl-urs-12-en-28-oic acid & 0.93 \\
\hline 3-Acetoxyimino-urs-12-en-28-oic acid & 24.16 \\
\hline $\begin{array}{l}\text { N-(3 } \beta \text {-Acetoxy-urs-12-en-28-oyl)-11- } \\
\text { aminoundecanoic acid methyl ester }\end{array}$ & $>500$ \\
\hline $3 \beta$-Acetoxy-urs-12-en-28-carboxamide & 105.25 \\
\hline $\begin{array}{l}\mathrm{N}-(3 \beta \text {-Hydroxy-urs-12-ene-28-oyl)-11-amino } \\
\text { undecanoic acid }\end{array}$ & 85.05 \\
\hline $3 \beta$-hydroxy-urs-12-ene-28-carboxamide & 27.96 \\
\hline $3 \beta$-phthaloyl-urs-12-en-28-carboxamide & 6.35 \\
\hline Ursolic acid & 3.25 \\
\hline Acarbose & 135.70 \\
\hline
\end{tabular}

Almost derivatives of ursolic acid exhibited inhibitory against $\alpha$-glucosidase better than acarbose, except compound N-(3 $\beta$-Acetoxy-urs-12-en-28-oyl)-11-aminoundecanoic acid methyl ester. Especially, derivative $3 \beta$-phthaloyl-urs-12-en-28-oic acid is 3.5 times more active than its lead compound (Table 3). This result has been similar with the recent report of $\mathrm{Wu}$ et al [23].

\section{Effects of ursolic acid on alloxan-induced diabetic mice in vivo}

As shown in the Table 5, the blood glucose level on alloxan-induced diabetic mice after oral administration of graded doses of $200 \mathrm{mg}$ and $300 \mathrm{mg}$ of ursolic acid $/ \mathrm{kg} /$ day. The results showed that alloxan-induced mice after oral administration of graded doses of ursolic acid with the dose as 200 and $300 \mathrm{mg} / \mathrm{kg} / \mathrm{day}$, the blood glucose level were reduced respectively $19.26 \%$ and $25.10 \%$ to compare with not drinking ursolic acid (day 0). Meanwhile, the blood glucose concentration of the control group was increased $26.21 \%$ comparing with before experiment. This showed that after 9 days alloxan-induced mice oral ursolic acid with doses of $200 \mathrm{mg}$ and $300 \mathrm{mg} / \mathrm{kg} / \mathrm{day}$, the serum glucose level was reduced $45.75 \%$ and $51.31 \%$ compared with the control group (Table 4 ). 
Table 4. Hypoglymecic effect of ursolic acid isolated from C. roseus on alloxan-induced diabetic mice.

\begin{tabular}{ccccc}
\hline Group & Day 0 & Day 10th & $\begin{array}{c}\text { \% change } \\
\text { compared with } \\
\text { day 0 }\end{array}$ & $\begin{array}{c}\text { Percentage } \\
\text { decrease } \\
\text { compared with } \\
\text { group 1 }\end{array}$ \\
Group 1 & $16,70 \pm 5,74$ & $22,63 \pm 4,03$ & $\uparrow 26,21$ & - \\
Group 2 & $16,57 \pm 3,88$ & $14,73 \pm 5,39^{*}$ & $\downarrow 11,07$ & 37,28 \\
Group 3 & $16,18 \pm 3,27$ & $13,02 \pm 4,08^{*}$ & $\downarrow 19,54$ & 45,75 \\
Group 4 & $16,73 \pm 2,05$ & $12,53 \pm 1,91^{*}$ & $\downarrow 25,10$ & 51,31 \\
\hline$p<0.05:$ Statistical significance versus diabetes control & &
\end{tabular}

The earlier reports revealed that the oral administration of leaves juice of $C$. roseus in healthy and alloxan-induced diabetic rabbits showed a significant antidiabetic activity and it had a more prolonged effect at $1.0 \mathrm{~mL} / \mathrm{kg}$ than the dlibencalmide dose at $40 \mu \mathrm{g} / \mathrm{kg}$ in the period of $18-24 \mathrm{~h}$ after treatment [11]. Another research by oral administrating of aquenous extract of C. roseus at a dose of $250 \mathrm{mg}, 350 \mathrm{mg}$, and $450 \mathrm{mg} / \mathrm{kg}$ body weight for 30 days to diabetic rats led to significant reduction in blood glucose, reduction in lipid profile and also prevented a decrease in body weight [12].Also, Qi et al [16] reported that ursolic acid is able to significantly relieve renal damage in mice with diabetic nephropathy induced by alloxan which may be involved in decreasing blood glucose level.

\section{CONCLUSIONS}

In conclusions, numberous pharmacological works and traditional usage have proved the high medicial properties of $C$. roseus. In this study, ursolic acid along with 7 compounds isolated from the whole plant of C.roseus have anti-glucosidase activity. Compounds 3, 4 and $\mathbf{5}$ have shown inhibitory $\alpha$-glucosidase better than acarbose. Especially, ursolic acid (5) is shown the highest activity against $\alpha$-glucosidase in vitro with $\mathrm{IC}_{50}$ value by $3.83 \mu \mathrm{g} / \mathrm{mL}$. While, the derivative $3 \beta$ phthaloyl-urs-12-en-28-oic acid has shown the greatest inhibition 3.5 times more than its lead compound. This study has confirmed that the main component with anti-hyperglycemia of $C$. roseus growing in Vietnam.

\section{Acknowledgement}

This research was supported by Vietnam Academy Science and Technology (VAST) of Vietnam (code: VAST04.06/13-14).

\section{References}

[1] N.H.Admad, R.A. Rahim, I. Mat. Catharenthus roseus aqueous extract is cytotoxic to Jurkat leukaemic T-cells but induces the proliferation of normal peripheral blood mononuclear cells. Trop Life Sci Res. 21 (2010) 101-113.

[2] F. Afrapoli-Moradi, B. Asghari, S.Saeidnia, Y. Ajani, M. Mirjai, M. Malmir, R.D. Bazaz, A.Hadjakhoondi, P. Salehi, M. Hamburger, N. Yassa. In vitro $\alpha$-glucosidase inhibitory activity of phenolic constituents from aerial parts of Polygonum hyrcanicum. Daru J Pharm Sci. 20 (2012)37.

[3] L.Almagro, F.P. Francisco, M.A. Pedreno. Indole Alkaloids from Catharanthus roseus: Bioproduction and their effect on human health. Molecules. 20 (2015) 2973-3000. 
[4] D.T. Dam. The effects of treating hypoglycemia and diabetes of some medicinal plants. J Pharm. 6 (2012) 430.

[5] I.D.F (International Diabetes Federation). Web: https: //www.idf.org/membership/wp/vietnam (2014; latest accession June, 2015).

[6] P.H. Ho. Flora of Vietnam. Ho Chi Minh City: Youth Publishing House, 2000, 512 p.

[7] W. Kang, Y. Song, X. Gu. $\alpha$-glucosidase inhibitory in vitro and antidiabetic activity in vivo of Osmanthus fragrans. J Med Plant Res. 6 (2012) 2850-2856.

[8] Y.P. Keepers, P.E. Pizao, G.J. Peter, J.V. Ark-Otte, B. Winograd, H.M. Pinedo. Comparison of the sulforhodamine B protein and tetrazolium (MTT) assays for in vitro chemosensitivity testing. Eur. J. Cancer. 27 (1991) 897-900.

[9] R.J. Marles, N.R. Farnsworth. Antidiabetic plants and their active constitutents. Phytomedicine. 2 (1995)137-189.

[10] A. Monks, D. Scudiero, P. Skehan, R. Shoemaker, K. Paull, D. Vistica, C. Hose, J.Langley, P.Cronise, A. Vaigro-Wolff, M. Gray-Goodrich, H. Campbell, J. Mayo, M. Boyd. Feasibility of a high-flux anticancer drug screen using a diverse panel of cultured human tumor cell lines. $\mathrm{J}$ Natl Cancer Inst. 83 (1991)757-766.

[11] S.Nammi, M.K. Boini, S.D. Lodagala, R. Babu, R.B.S. Behara. The juice of fresh leaves of Catharenthus roseus Linn. Reduces blood glucose in normal and alloxan diabetic rabbits. BMC Complement Altern Med. 3 (2003) 4.

[12] A. Natarajian, K.S.Z. Ahmed, S. Sundaresan, A. Sivaraj, K. Devi, B.S. Kumar. Effect aqueous flower extract of Cathanranthus roseus on alloxan induced diabetes in male albino rats. Inter $\mathrm{J}$ Pharm Sci Drug Res. 4 (2012) 150-153.

[13] L. Li, X.D. Zhang, Y.W. Song, J.W. Liu. A microplate-based screening method for $\alpha$ glucosidase inhibitors. Chin J Clin Pharmacol Ther. 10 (2005) 1128-1134.

[14] S.C. Ohadoma, H.U. Michael. Effects of co-administration of methanol leaf extract of Catharanthus roseus on the hypoglycemic activity of metformin and glibenclamide in rats. Asian Pac J Trop Med. 4 (2014) 475-477.

[15] M.M. Rahman, M.N. Hasan, A.K. Das, M.T. Hossain, R. Jahan, M.A. Khatun, M. Rahmatullah. Effect of Delonix regia leaf extract on glucose tolerance in glucose induced hyperglycemic mice. Afr Tradit Complement Altern Med. 8 (2011) 34-36.

[16] M.Y. Qi, J.J. Yang, B. Zhou, D.Y. Pan, X. Sun. Study on the protective effect of ursolic acid on alloxan-induced diabetic renal injury and its underlying mechanisms. Zhonqquo Ying Yong Sheng Li Xue Za Zhi. 30 (2014) 445-448 (Chinese with abstract in English).

[17] M.J. Siddiqui, Z. Ismail, A.F.A. Aisha, A.M.S. Abdul Majid. Cytotoxicity activity of Catharanthus roseus crude extracts and pure compounds against human coorctal carcinoma cell line. Inter J Pharm. 5 (2010) 43-47.

[18] B. Suparb, and P. Amorn. Chemical constituents of the roots of Bridelia tomentosa Bl. J. Sci. Soc. Thailand. 17 (1991) 61-69.

[19] N.T. Tam, D.D. Thien, T.V. Sung. Further chemical constituents of Catharanthus rosues (L.) G. Don collected from Binh Dinh province, Vietnam.Vietnamese J Chem. 52 (2014) 626-628.

[20] D.D. Thien, N.T. Tam, D.G. Thien, N.T.H. Anh, T.V. Sung.Synthesis and cytotoxic activity of ursolic acid derivatives. Zeitschrift für Naturforschung B. 68b (2013) 201-206.

[21] S.H. Tiong, C.Y. Looi, A. Arya, W.F. Wong, H. Hazni, M.R. Mustafa, K. Awang. Vindogentianine, a hypoglycemic alkaloid from Catharenthus roseus (L.) G.Don (Apocynaceae). Fitoterapia. 102 (2015)182-188. 
[22] R. Van der Heijden, D.I. Jacobs, W. Snoeijer, D. Hallard, R. Verpoorte. The Catharanthus alkaloids: Pharmacognosy and biotechnology. Curr Med Chem. 11 (2004)1241-1253.

[23] P.P. Wu, K. Zhang, Y.J. Lu, P. He, S.Q. Zhao. In vitro and in vivo evaluation of the antidiabetic activity of ursolic acid derivatives. Eur J Med Chem. 80 (2014) 502-508.

[24] R. Yanarday, H. Colac. Effect chard (Beta vulgaris L. varcicla) on blood glucose level in normal and alloxan induced diabetic rabiit. J. Ethnopharm. 4 (1998) 309-311. 